\title{
Massive obscure-overt upper gastrointestinal bleeding secondary to hemobilia
}

Hemobilia is an uncommon cause of upper gastrointestinal bleeding $[1,2]$. Here we present two cases of obscure-overt upper gastrointestinal bleeding that were caused by hemobilia from a hepatic artery.

Case 1: A 74-year-old man presented with hematemesis associated with jaundice and fever, on a background of advanced gallbladder cancer with metastases that had been treated palliatively with placement of self-expandable metallic stent (SEMS) for relief of obstructive jaundice. Urgent esophagogastroduodenoscopy (EGD) was unremarkable. A 64-multislice computed tomography (MSCT) scan of the abdomen did not reveal the source of bleeding. The SEMS was noted to be abutting the right hepatic artery ( $\bullet$ Fig. 1 a). However, the patient experienced rebleeding and urgent mesenteric/hepatic angiography revealed a constriction in the right hepatic artery close to the SEMS. At the point of constriction, there was extravasation of contrast into the lumen of the SEMS. The constricted segment of the artery was embolized with the help of VortX (Boston Scientific International, La Garenne Colombes, France) coils and gel foam. The postembolization check angiogram confirmed hemostasis and occlusion of the artery ( Fig. $\mathbf{1}$ b, c).

Case 2: A 53-year-old woman presented with melena, jaundice, and pain in the right hypochondrium, on a background of recurrent pyogenic cholangitis (RPC) that had been treated with partial hepatectomy and cholecystectomy with biliary bypass. In view of the presence of the Quincke triad, hemobilia was suspected. Urgent EGD revealed blood oozing from ampulla of Vater ( Fig. 2a). Part of the duodenum was indented, presumably by the distended blood-filled common bile duct. Urgent 64-MSCT of the abdomen showed a pseudoaneurysm in the left hepatic artery as well as exudation of intravenous contrast in the intrahepatic and extrahepatic biliary tree. Immediate mesenteric angiography confirmed hemobilia from the left hepatic artery pseudoaneurysm, and embolization resulted in successful hemostasis ( $\bullet$ Fig. $\mathbf{2 b}, \mathbf{c}$ ).
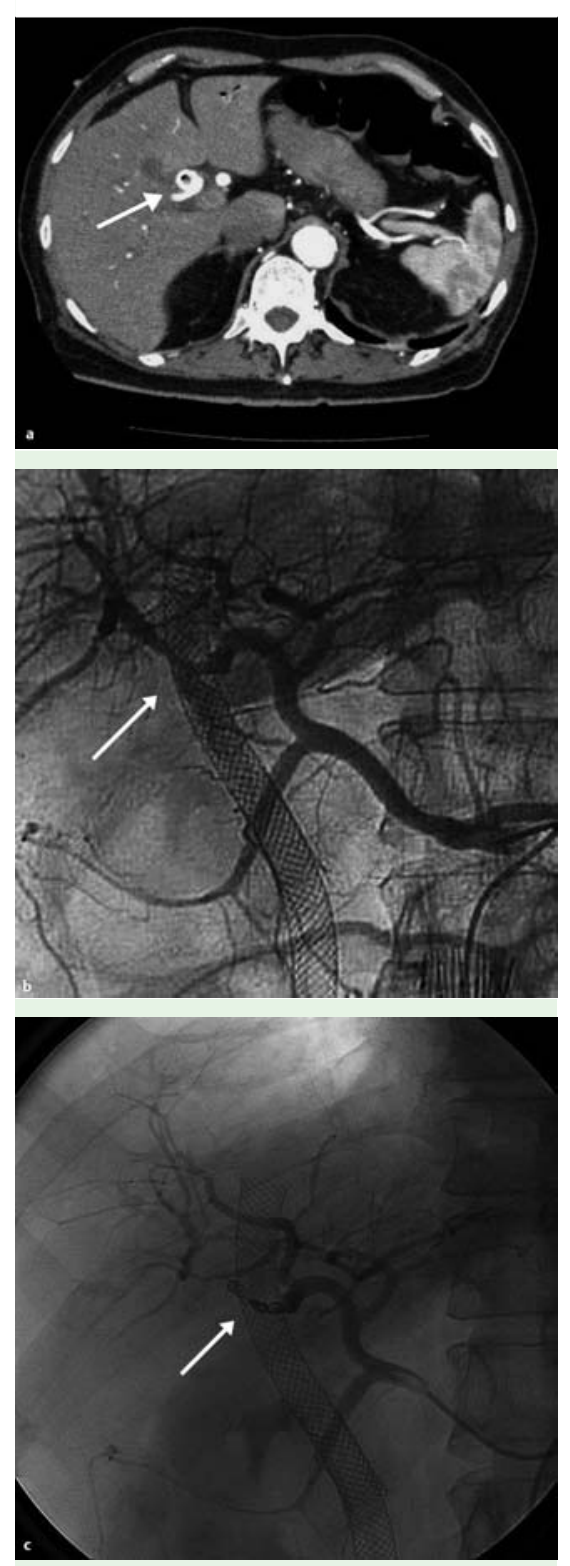

Fig. 1 a A 64-multislice computed tomography (MSCT) scan showing the self-expanding metallic stent (SEMS) abutting the right hepatic artery. $\mathbf{b}$ Angiogram showing constriction of the right hepatic artery and extravasation of the contrast into the SEMS lumen. c Successful deployment of the VortX coils resulting in hemostasis.

Hemobilia is an uncommon cause of upper gastrointestinal bleeding [1,2]. In cases of obscure-overt upper gastrointestinal bleeding in which risk factors for hemobilia are present, mesenteric angiogra-
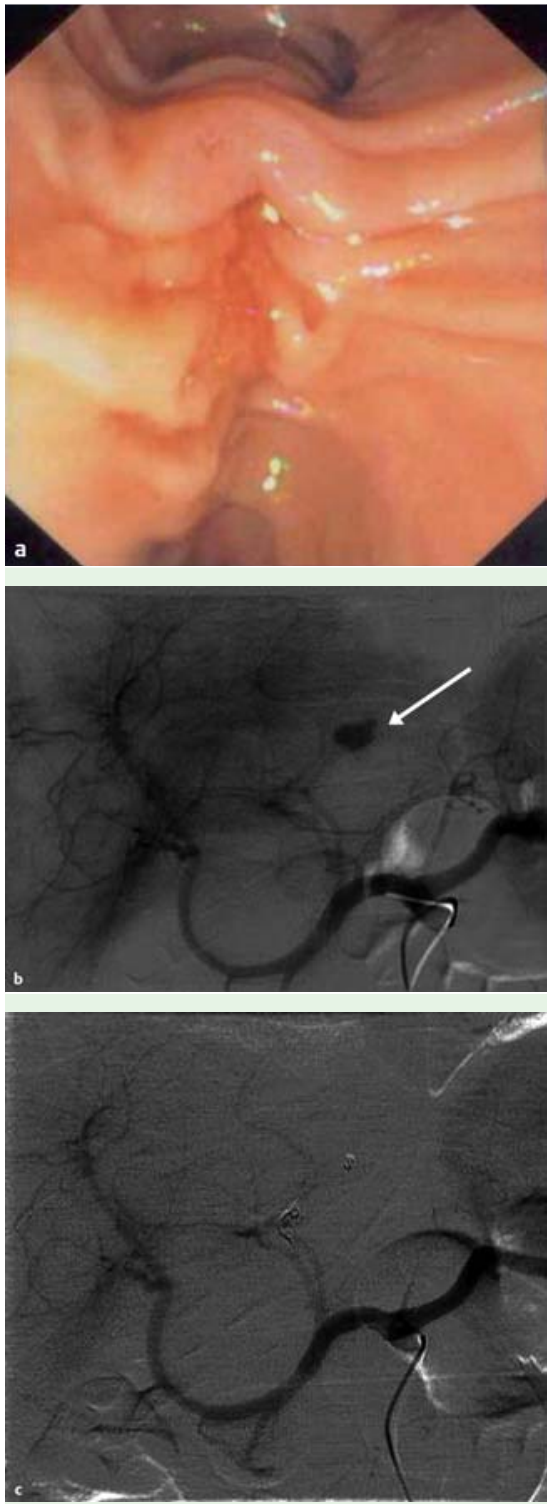

Fig. 2 a Hemobilia seen on esophagogastroduodenoscopy. b Mesenteric angiogram showing hemobilia from the left hepatic artery aneurysm. c Embolization resulting in successful hemostasis.

phy seems to be the most appropriate second-line investigation if the initial endoscopy is negative [3]. There is also the added advantage that hemostasis can be achieved via embolization at the time of the procedure [1-5].

Endoscopy_UCTN_Code_CPL_1AK_2AD 
P. S. $\operatorname{Tan}^{1 *}$, E. K. Teo ${ }^{1 *}$, K. M. Fock ${ }^{1}$,

T. L. Ang', J. Y. L. Tan' ${ }^{1}$ A. G. S. Tan ${ }^{2}$

1 Division of Gastroenterology, Department of Medicine, Changi General Hospital, Singapore

2 Department of Radiology, Changi General Hospital, Singapore

\section{References}

1 Merrell SW, Schneider PD. Hemobilia - evolution of current diagnosis and treatment. West J Med 1991; 155: 621 - 625

2 Bloechle C, Izbicki JR, Rashed MY et al. Hemobilia: presentation, diagnosis, and management. Am J Gastroenterol 1994; 89: 1537 1540

3 Xu ZB, Zhou XY, Peng ZY et al. Evaluation of selective hepatic angiography and embolisation in patients with massive hemobilia. Hepatobiliary Pancreat Dis Int 2005; 4: 254-258

4 Curet P, Baumer R, Roche A et al. Hepatic hemobilia of traumatic or iatrogenic origin: Recent advances in diagnosis and therapy, review of the literature from 1976 to 1981. World J Surg 1984; 8: 2-8

5 Sokiranski R, Rilinger $N$, Brado $M$ et al. Interventional treatment of hemobilia. Rofo 1997; 166: 417-420
Bibliography

DOI $10.1055 / \mathrm{s}-0029-1215072$

Endoscopy 2009; 41: E294 -E295

(c) Georg Thieme Verlag KG Stuttgart · New York . ISSN 0013-726X

\section{Corresponding author}

\section{Dr. P. S. Tan}

Division of Gastroenterology,

Department of Medicine, Changi General Hospital 2 Simei Street 3

Singapore 529889

Fax: +(65) 68503558

Poh_Seng_Tan@cgh.com.sg

${ }^{*}$ P. S. Tan und E. K. Teo are both authors for this manuscript. It is given under the corresponding author's details. 